\title{
Vitamin D Deficiency and Increased Risk of Bladder Carcinoma: A Meta-Analysis
}

\author{
Hui Zhang ${ }^{a}$ Hui Zhang ${ }^{b}$ Xiuhua Wen ${ }^{a}$ Yonggang Zhang ${ }^{a} \quad$ Xueli Weia \\ Taiyang Liu ${ }^{a}$
}

aDepartment of Urology, Central Hospital of Zhumadian City, Zhumadian City, ${ }^{b} B a s i c$ Medicine of Nurse Department, Huanghuai College, Zhumadian City, China

\section{Key Words}

Vitamin D • Bladder carcinoma $\cdot$ Meta-analysis

\begin{abstract}
Background/Aims: Vitamin D status in relation to bladder carcinoma risk was still inconsistent. This study was carried out to evaluate the relationship between vitamin D status and bladder carcinoma risk through a meta-analysis approach. Methods: Pubmed, Web of Science, CNKI, and Embase were searched systemically to find eligible studies from the earliest available date to April 16, 2015. The search terms "vitamin D", "25-hydroxyvitamin D", "bladder cancer" or "bladder carcinoma" were used to retrieve relevant studies. The exposure of interest was intake of vitamin $\mathrm{D}$ or serum vitamin $\mathrm{D}$ levels, and the outcome of interest was bladder carcinoma incidence or mortality. The pooled risk ratio (RR) values and their 95\%CIs were calculated through meta-analysis. Results: Seven studies with a total of 62,141 participants met the inclusion criteria and were finally included into the meta-analysis. There was no heterogeneity among those included studies $\left(I^{2}=0 \%, P=0.53\right)$. The pooled $R R$ of bladder carcinoma for the lowest category versus the highest category of vitamin D was 1.34 (95\% CI 1.17-1.53, P < 0.0001 ). Sensitivity analysis by omitting one study by turns showed all the pooled RRs were statistically significant. Meta-analysis of 5 studies reporting outcomes of serum vitamin D levels also showed that the low serum vitamin $D$ level was associated with increased risk of bladder carcinoma ( $R R=1.32,95 \% C I 1.15-1.52, P=0.0001)$. No obvious risk of publication bias was observed. Conclusion: Vitamin D deficiency is associated with increased risk of bladder carcinoma in present study.

Copyright $\odot 2015$ S. Karger AG, Basel
\end{abstract}

\section{Introduction}

Bladder carcinoma is a common malignant disease and it causes approximately 150,000 deaths per year worldwide [1]. In United States, bladder carcinoma is the fourth most commonly diagnosed cancer in men and eighth leading cause of cancer-related death [2]. It's has been well known that bladder carcinoma is a heterogeneous disease and $30 \%$ 
of patients have muscle-invasive disease and have bad prognosis [1]. Though many factors have been identified as risk factors of bladder carcinoma, such as age, smoking, diabetes, and obesity, the pathogenesis of bladder carcinoma is still not well understood [3-5]. Vitamin $\mathrm{D}$ is a main and important precursor to the potent steroid hormone calcitriol, and it has widespread and vital actions throughout the body [6, 7]. Previous studies have suggested that vitamin D is involved in the numerous cellular pathways and has some possibility of preventing cancer $[7,8]$. Epidemiological studies have proven a beneficial role of vitamin $D$ in preventing colorectal cancer and breast caner $[9,10]$. 25-hydroxyvitamin $\mathrm{D}(25(\mathrm{OH}) \mathrm{D})$ is the main existence form of vitamin D in human body. Previous studies have shown that $25(\mathrm{OH})$ D has antitumor activities by mediating cell differentiation and apoptosis, and inhibiting angiogenesis and metastasis [7]. Previous studies also have suggested that vitamin D can inhibit proliferation and induce apoptosis in human bladder tumor cells in vitro, and may have therapeutic potential in bladder cancer [11, 12]. Several epidemiological studies were published to assess the relationship between vitamin D status and bladder carcinoma risk, but failed to find consistent results $[9,13-16]$. Thus, vitamin D status in relation to bladder carcinoma risk was still unclear. This study was carried out to evaluate the relationship between vitamin D status and bladder carcinoma risk through a meta-analysis approach.

\section{Materials and Methods}

\section{Search strategy and study selection}

Pubmed, Web of Science, CNKI, and Embase were searched systemically to find eligible studies on the relationship between vitamin D status and bladder carcinoma risk. The searching time was from the earliest available date to September 6, 2014. An updated literature was performed on April 16, 2015. We also searched unpublished data through Google Scholar. There was no language in our literature search. The following search terms were used: ("vitamin D" or "25-hydroxyvitamin D") and ("bladder cancer" or "bladder" or "bladder carcinoma"). The references of eligible studies or relevant reviews were also searched for other studies.

To be included into the meta-analysis, studies must meet the following criteria: (1) Cohort studies, nested case-control studies, or retrospective case-control studies; (2) The exposure of interest was intake of vitamin D or serum vitamin D levels; (3) The outcome of interest was bladder carcinoma incidence or mortality; (4) The study must report data to calculate risk estimates (Risk ratio [RR], Relative risk [RR], or Odds ratio [OR]) and their $95 \%$ CIs for bladder carcinoma. Studies using animal models, case-only studies, or reviews were all excluded. For studies with overlapping data, only the study with the largest data set was included.

\section{Data extraction and quality assessment}

Data extraction was performed independently by two reviewers, and differences were resolved by consensus. We extracted the following data from each included study using standardized forms: first author name, publication year, study design, country of participants, mean age, gender, categories of vitamin D, and risk estimates according to vitamin D status. The methodological quality of the studies was assessed using the 9-star Newcastle-Ottawa Scale (NOS) for cohort studies [17]. According to the NOS scale, methodological quality of included studies in the meta-analysis was assessed according to three study components: selection of participants; comparability of participants; and ascertainment of outcomes. Studies with 6 or more stars were defined as high quality studies.

\section{Statistical analysis}

The RR with 95\% CI of the lowest category of serum vitamin D level or vitamin D intake for bladder carcinoma compared with the highest category group were calculated for each study, and were then pooled using meta-analysis. The heterogeneity was assessed using both chi-squared test and $\mathrm{I}^{2}$ method $[18,19]$. When $P$ value for chi-squared test was less than 0.10 or $I^{2}$ was more than $50 \%$, there was obvious heterogeneity among those included studies, and random-effect model was used to pool data [20]. If there was no obvious heterogeneity among those included studies, a fixed-effect model was used to pool data 
[21]. Subgroup analysis was performed by study design or study quality. Sensitivity analysis was performed by omitting one study by turns to test the changes of pooled RRs. Potential risk of publication bias was estimated by inspection of funnel plot. Publication bias was also assessed by Egger's test. Stata version 12 (Stata Corporation, USA) was used for statistical analyses.

\section{Results}

Literature search and study characteristics

A total of 156 individual abstracts for potential studies were identified through literature search. 147 abstracts were excluded because they were irrelevant to the current meta-analysis, and 9 studies were left and were assessed by reading full-text [13-16, 22-26]. One study was excluded for lack of usable data [22], and one was excluded for containing overlapping data [26]. Thus, seven studies met the inclusion criteria and were finally included into the meta-analysis [13-16, 23-25]. Those 7 studies contained with a total of 62,141 participants, and were published from 2006 to 2013 [13-16, 23-25]. Among those 7 studies, two were prospective cohort studies $[13,16]$, two were nested case-control studies $[14,25]$, and the other 3 studies were case-control studies [15, 23, 24]. All studies reported adjusted risk estimates, but the confounding factors were different among those studies [13-16, 23-25]. Five studies assessed the relationship between serum vitamin D levels and bladder carcinoma risk [13-16, 25], and the other two studies assessed the relationship between dietary vitamin D intake and bladder carcinoma risk [23, 24]. According to NOS criteria, four studies had high quality $[13,14,16,25]$, while the other 3 studies didn't have high quality $[15,23,24]$.

\section{Meta-analysis}

There was no heterogeneity among those seven included studies $\left(\mathrm{I}^{2}=0 \%, \mathrm{P}=0.53\right)$. So the fixed-effect model was used to pool data. The pooled RR of bladder carcinoma for the lowest category versus the highest category of vitamin D was 1.34 (95\% CI 1.17-1.53, P <

\begin{tabular}{|c|c|c|c|c|c|c|}
\hline Study or Subgroup & log[Risk Ratio] & SE & Weight & $\begin{array}{l}\text { Risk Ratio } \\
\text { M. Fixed, } 95 \% \mathrm{CI}\end{array}$ & $\begin{array}{r}\text { Risk R } \\
\text { IV. Fixed. }\end{array}$ & $\begin{array}{l}\text { Ratio } \\
\text {.95\% CI }\end{array}$ \\
\hline Afzal (2013) & 0.2469 & 0.0962 & $49.8 \%$ & $1.28[1.06,1.55]$ & & - \\
\hline Amaral(2012) & 0.6043 & 0.2196 & $9.6 \%$ & $1.83[1.19,2.81]$ & & \\
\hline Brinkman $(2010)$ & 0.5446 & 0.3196 & $4.5 \%$ & $1.72[0.92,3.23]$ & & \\
\hline Erinkman(2011) & 0.2746 & 0.2452 & $7.7 \%$ & $1.32[0.81,2.13]$ & & \\
\hline Giovannucci (2006) & 0.1044 & 0.182 & $13.9 \%$ & $1.11[0.78,1.59]$ & & \\
\hline Mondul(2010) & 0.5481 & 0.2646 & $6.6 \%$ & $1.73[1.03,2.91]$ & & \\
\hline Mondul(2012) & 0.1655 & 0.241 & $7.9 \%$ & $1.18[0.74,1.89]$ & & \\
\hline Total (95\% Cl) & & & $100.0 \%$ & $1.34[1.17,1.53]$ & & \\
\hline \multicolumn{5}{|c|}{$\begin{array}{l}\text { Heterogeneity: } \mathrm{Ch}^{2}=5.15, \mathrm{df}=6(\mathrm{P}=0.53) ; \mathrm{I}^{2}=0 \% \\
\text { Test for overall effect: } Z=4.27(\mathrm{P}<0.0001)\end{array}$} & $\begin{array}{ccc}0.5 & 0.7 & 1 \\
\text { Favours low status }\end{array}$ & $\begin{array}{c}1.52 \\
\text { Favours high status }\end{array}$ \\
\hline
\end{tabular}

Fig. 1. Low vitamin D status was obviously associated with increased risk of bladder.

\begin{tabular}{|c|c|c|c|c|c|c|c|c|}
\hline Studv or Subgroup & log[Risk Ratio] & SE & Weight & $\begin{array}{c}\text { Risk Ratio } \\
\text { IN, Fixed, 95\% Cl }\end{array}$ & & $\begin{array}{r}\text { Risk F } \\
\text { IV, Fixed. }\end{array}$ & $\begin{array}{l}\text { Ratio } \\
\text { d, } 95 \% \mathrm{Cl}\end{array}$ & \\
\hline Afral(2013) & 0.2469 & 0.0962 & $56.7 \%$ & $1.28[1.06,1.55]$ & & & $-1-$ & \\
\hline Amaral(2012) & 0.6043 & 0.2196 & $10.9 \%$ & $1.83[1.19,2.81]$ & & & & \\
\hline Giovannucci (2006) & 0.1044 & 0.182 & $15.8 \%$ & $1.11[0.78,1.59]$ & & & & \\
\hline Mondul(2010) & 0.5481 & 0.2646 & $7.5 \%$ & $1.73[1.03,2.91]$ & & & & \\
\hline Mondul(2012) & 0.1655 & 0.241 & $9.0 \%$ & $1.18[0.74,1.89]$ & & & & \\
\hline Total $(95 \% \mathrm{Cl})$ & & & $100.0 \%$ & $1.32[1.15,1.52]$ & & & & \\
\hline \multicolumn{5}{|c|}{$\begin{array}{l}\text { Heterogeneity: Chi }=4.48, d f=4(P=0.34) ; i^{2}=11 \% \\
\text { Test for overall effect } Z=3.84(P=0.0001)\end{array}$} & 0.2 & $\begin{array}{c}0.5 \\
\text { Favours low status }\end{array}$ & 1 Favours high status & 5 \\
\hline
\end{tabular}

Fig. 2. Low serum vitamin D level was obviously associated with increased risk of bladder. 
Fig. 3. Funnel plot didn't show obvious risk of publication bias.

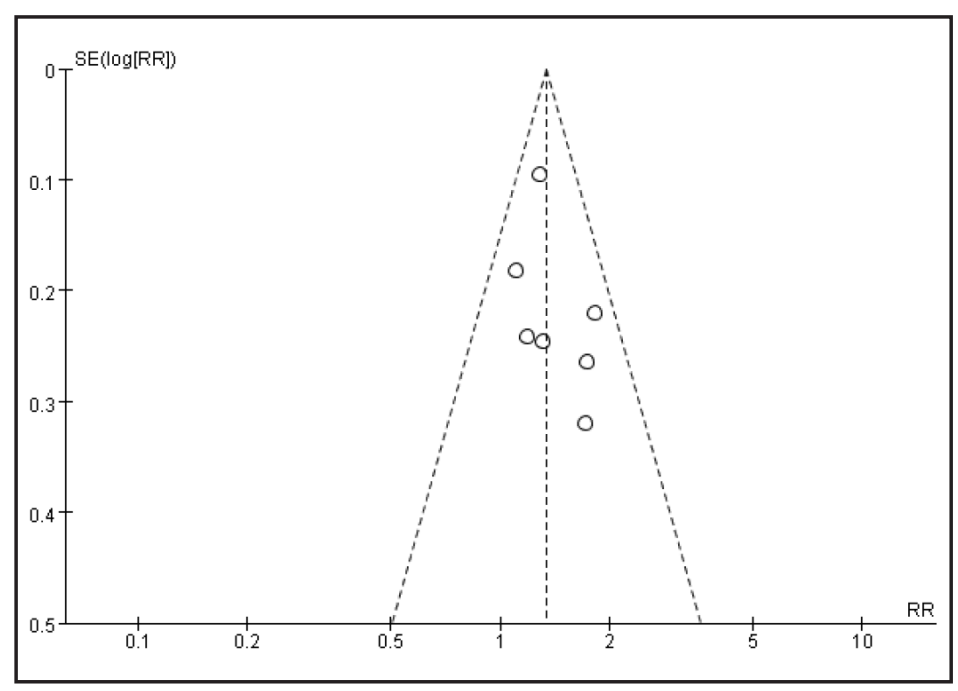

0.0001) (Fig. 1). Sensitivity analysis performed by omitting one study by turns showed all the pooled RRs were statistically significant.

Meta-analysis of those 5 studies on serum vitamin D levels also showed that low serum vitamin $\mathrm{D}$ level was associated with increased risk of bladder carcinoma ( $\mathrm{RR}=1.32,95 \% \mathrm{CI}$ 1.15-1.52, $\mathrm{P}=0.0001 ; \mathrm{I}^{2}=0 \%$ ) (Fig. 2). Meta-analysis of those two studies on dietary vitamin D intake showed that low vitamin D intake was marginally associated with increased risk of bladder carcinoma (RR = 1.45, 95\%CI 0.99-2.13, $\left.\mathrm{P}=0.05 ; \mathrm{I}^{2}=0 \%\right)$.

Subgroup analysis of studies with high quality showed that lowest vitamin D status was still associated with increased risk of bladder carcinoma $(\mathrm{RR}=1.27,95 \% \mathrm{CI} 1.09-1.48$, $\mathrm{P}=0.002 ; \mathrm{I}^{2}=0 \%$ ). Subgroup analysis of studies without high quality showed that lowest vitamin $\mathrm{D}$ status was also associated with increased risk of bladder carcinoma $(\mathrm{RR}=1.61$, 95\%CI 1.21-1.24, P = 0.001; $\mathrm{I}^{2}=0 \%$ ).

Potential risk of publication bias was estimated by inspection of funnel plot, and the funnel plot for meta-analysis of total 7 studies didn't suggest possible risk of publication bias in the meta-analysis (Fig. 3). In addition, the P value for Egger's test was more than 0.05, which showed no obvious risk of publication bias in the meta-analysis.

\section{Discussion}

There are accumulating results from epidemiological studies proving a beneficial role of vitamin D in preventing cancer risk $[9,10]$. Several epidemiological studies were published to assess the relationship between vitamin D status and bladder carcinoma risk, but failed to find consistent results [9, 13-16]. Currently, there is lack of good epidemiological evidence for the association between vitamin D deficiency and bladder carcinoma risk. We performed this meta-analysis to evaluate the relationship between vitamin D status and bladder carcinoma risk. Seven studies with a total of 62,141 participants met the inclusion criteria and were finally included into the meta-analysis [13-16, 23-25]. The findings suggest low serum vitamin D level is associated with increased risk of bladder carcinoma (Fig. 1, Fig. 2). Therefore, vitamin D deficiency is associated with increased risk of bladder carcinoma in present study.

This present meta-analysis is the first one on the association between vitamin D deficiency and bladder carcinoma risk. Though only 7 eligible studies were available and included into the meta-analysis, there was no between-study heterogeneity among those seven included studies $\left(\mathrm{I}^{2}=0 \%, \mathrm{P}=0.53\right)$, which could strengthen the evidence for the association between vitamin $\mathrm{D}$ deficiency and bladder carcinoma risk. In addition, subgroup 
analysis and sensitivity analysis further suggested that the pooled risk estimate of bladder carcinoma was consistent in the meta-analysis. Thus, the meta-analysis provided strong evidence for the association between vitamin D deficiency and bladder carcinoma risk.

Previous studies suggest that vitamin D can inhibit proliferation and induce apoptosis in human bladder tumor cells in vitro $[11,12,27]$. The findings from the meta-analysis provide strong epidemiological evidence for the important role of vitamin D in bladder carcinogenesis. There are several studies published to assess the role of vitamin D deficiency on the prognosis of cancer patients, and vitamin D deficiency may decrease the overall survival of cancer patients, such as colorectal cancer [28]. However, there is no study performed to assess the relation of vitamin D deficiency with prognosis of bladder carcinoma patients, and there is still lack of clinical evidence. Thus, further studies are needed to further assess the prognostic role of vitamin D deficiency in patients with bladder carcinoma.

25(OH)D is the main existence form of vitamin D in human body. Previous studies have shown that 25(OH)D has antitumor activities by mediating cell differentiation and apoptosis, and inhibiting angiogenesis and metastasis $[7,29,30]$. Previous studies have shown that vitamin D deficiency is associated with increased risk of cancers, such as lung cancer [31, 32]. Previous studies also have suggested that vitamin D can inhibit proliferation and induce apoptosis in human bladder tumor cells in vitro, and may have therapeutic potential in bladder cancer $[11,12]$. Therefore, high level of vitamin D in human body has the effect of preventing or delaying the onset of bladder carcinoma. On the contrary, individuals with vitamin D deficiency thus have higher risk of bladder carcinoma. Some studies also have found that vitamin D receptor (VDR) are also involved in the function of vitamin D, but there is no relevant study to assess the roles of VDR in bladder cancer [33, 34]. Future studies may further investigate the roles of VDR in bladder cancer.

The findings in the meta-analysis may be interpreted with caution owing to several limitations. First, the definition criteria for vitamin D deficiency or low vitamin D status were obvious different between those included studies, which may decrease the accuracy of the pooled risk estimates. In addition, vitamin D deficiency may occur in several quartiles but many of the studies were of those in the lowest grouping, which may lead to certain risk of bias. More studies with strict definition of vitamin D deficiency are needed in the future. Second, there were only two studies with prospective cohort design, and four studies with high quality. To decrease risk of bias, more studies with prospective cohort design are needed. Finally, though the funnel plot for meta-analysis of total 7 studies didn't suggest possible risk of publication bias in the meta-analysis, the power to detect publication bias for funnel plot may decrease when there were only 7 studies. Thus, we were unable to exclude all possibility of publication bias in the meta-analysis.

In conclusion, this meta-analysis shows that vitamin D deficiency is associated with increased risk of bladder carcinoma, and it's a risk factor for bladder carcinoma. Further prospective cohort studies with large number of participants are needed to further assess the risk of bladder carcinoma in individuals with vitamin D deficiency. The prognostic role of vitamin D deficiency in bladder carcinoma is also interesting and needs future studies.

\section{Disclosure Statement}

None.

\section{References}

1 Kaufman DS, Shipley WU, Feldman AS: Bladder cancer. Lancet 2009;374:239-249.

- Siegel R, Ma J, Zou Z, Jemal A: Cancer statistics, 2014. CA Cancer J Clin 2014;64:9-29.

-3 Mitra AP, Cote RJ: Molecular screening for bladder cancer: Progress and potential. Nat Rev Urol 2010;7:1120. 


\section{Cellular Physiology Cell Physiol Biochem 2015;37:1686-1692 \begin{tabular}{l|l|l} 
DOI: 10.1159/000438534 & (C) 2015 S. Karger AG, Basel
\end{tabular} and Biochemistry Publishea onine: November 09, 2015

4 Cancer Genome Atlas Research Network: Comprehensive molecular characterization of urothelial bladder carcinoma. Nature 2014;507:315-322.

5 Freedman ND, Silverman DT, Hollenbeck AR, Schatzkin A, Abnet CC: Association between smoking and risk of bladder cancer among men and women. JAMA 2011;306:737-745.

6 Hart PH, Gorman S, Finlay-Jones JJ: Modulation of the immune system by uv radiation: More than just the effects of vitamin d? Nat Rev Immunol 2011;11:584-596.

-7 Feldman D, Krishnan AV, Swami S, Giovannucci E, Feldman BJ: The role of vitamin d in reducing cancer risk and progression. Nat Rev Cancer 2014;14:342-357.

-8 Theodoratou E, Tzoulaki I, Zgaga L, Ioannidis JP: Vitamin d and multiple health outcomes: Umbrella review of systematic reviews and meta-analyses of observational studies and randomised trials. BMJ 2014;348:g2035.

-9 Ma Y, Zhang P, Wang F, Yang J, Liu Z, Qin H: Association between vitamin d and risk of colorectal cancer: A systematic review of prospective studies. J Clin Oncol 2011;29:3775-3782.

10 Kim Y, Je Y: Vitamin d intake, blood 25(oh)d levels, and breast cancer risk or mortality: A meta-analysis. Br J Cancer 2014;110:2772-2784.

11 Konety BR, Lavelle JP, Pirtskalaishvili G, Dhir R, Meyers SA, Nguyen TS, Hershberger P, Shurin MR, Johnson CS, Trump DL, Zeidel ML, Getzenberg RH: Effects of vitamin d (calcitriol) on transitional cell carcinoma of the bladder in vitro and in vivo. J Urol 2001;165:253-258.

-12 Sahin MO, Canda AE, Yorukoglu K, Mungan MU, Sade M, Kirkali Z: 1,25 dihydroxyvitamin d(3) receptor expression in superficial transitional cell carcinoma of the bladder: A possible prognostic factor? Eur Urol 2005;47:52-57.

13 Giovannucci E, Liu Y, Rimm EB, Hollis BW, Fuchs CS, Stampfer MJ, Willett WC: Prospective study of predictors of vitamin d status and cancer incidence and mortality in men. J Natl Cancer Inst 2006;98:451459.

14 Mondul AM, Weinstein SJ, Mannisto S, Snyder K, Horst RL, Virtamo J, Albanes D: Serum vitamin d and risk of bladder cancer. Cancer Res 2010;70:9218-9223.

-15 Amaral AF, Mendez-Pertuz M, Munoz A, Silverman DT, Allory Y, Kogevinas M, Lloreta J, Rothman N, Carrato A, Rivas del Fresno M, Real FX, Malats N: Plasma 25-hydroxyvitamin d(3) and bladder cancer risk according to tumor stage and fgfr3 status: A mechanism-based epidemiological study. J Natl Cancer Inst 2012;104:1897-1904.

16 Afzal S, Bojesen SE, Nordestgaard BG: Low plasma 25-hydroxyvitamin d and risk of tobacco-related cancer. Clin Chem 2013;59:771-780.

17 Wells G, Shea B, O'connell D, Peterson J, Welch V, Losos M, Tugwell P: The newcastle-ottawa scale (nos) for assessing the quality of nonrandomised studies in meta-analyses. Ottawa Health Research Institute Web site, 2014, http://www.ohri.ca/programs/clinical_epidemiology/oxford.asp

18 Cochran WG: The combination of estimates from different experiments. Biometrics 1954;10:101-129.

19 Higgins JP, Thompson SG, Deeks JJ, Altman DG: Measuring inconsistency in meta-analyses. BMJ 2003;327:557-560.

20 DerSimonian R, Laird N: Meta-analysis in clinical trials. Control Clin Trials 1986;7:177-188.

-21 Mantel N, Haenszel W: Statistical aspects of the analysis of data from retrospective studies of disease. J Natl Cancer Inst 1959;22:719-748.

22 Vena JE, Graham S, Freudenheim J, Marshall J, Zielezny M, Swanson M, Sufrin G: Diet in the epidemiology of bladder cancer in western new york. Nutr Cancer 1992;18:255-264.

-23 Brinkman MT, Karagas MR, Zens MS, Schned A, Reulen RC, Zeegers MP: Minerals and vitamins and the risk of bladder cancer: Results from the new hampshire study. Cancer Causes Control 2010;21:609-619.

24 Brinkman MT, Buntinx F, Kellen E, Dagnelie PC, Van Dongen MC, Muls E, Zeegers MP: Dietary intake of micronutrients and the risk of developing bladder cancer: Results from the belgian case-control study on bladder cancer risk. Cancer Causes Control 2011;22:469-478.

25 Mondul AM, Weinstein SJ, Horst RL, Purdue M, Albanes D: Serum vitamin d and risk of bladder cancer in the prostate, lung, colorectal, and ovarian (plco) cancer screening trial. Cancer Epidemiol Biomarkers Prev 2012;21:1222-1225.

-26 Mondul AM, Weinstein SJ, Virtamo J, Albanes D: Influence of vitamin d binding protein on the association between circulating vitamin $d$ and risk of bladder cancer. Br J Cancer 2012;107:1589-1594. 


\section{Cellular Physiology Cell Physiol Biochem 2015;37:1686-1692

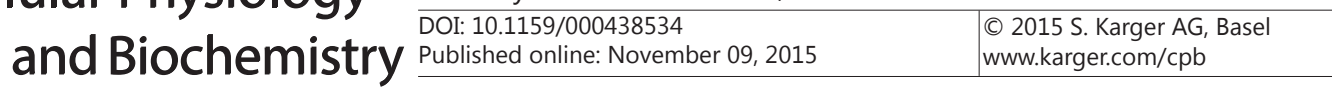 \\ Zhang et al.: Vitamin D and Bladder Cancer}

27 Hsu JW, Yin PN, Wood R, Messing J, Messing E, Lee YF: 1 alpha, 25-dihydroxylvitamin d3 promotes bacillus calmette-guerin immunotherapy of bladder cancer. Oncotarget 2013;4:2397-2406.

28 Zgaga L, Theodoratou E, Farrington SM, Din FV, Ooi LY, Glodzik D, Johnston S, Tenesa A, Campbell H, Dunlop MG: Plasma vitamin d concentration influences survival outcome after a diagnosis of colorectal cancer. J Clin Oncol 2014;32:2430-2439.

29 Autier P, Boniol M, Pizot C, Mullie P: Vitamin d status and ill health: A systematic review. Lancet Diabetes Endocrinol 2014;2:76-89.

-30 Bolland MJ, Grey A, Gamble GD, Reid IR: The effect of vitamin d supplementation on skeletal, vascular, or cancer outcomes: A trial sequential meta-analysis. Lancet Diabetes Endocrinol 2014;2:307-320.

31 Zhang L, Wang S, Che X, Li X: Vitamin d and lung cancer risk: a comprehensive review and meta-analysis. Cell Physiol Biochem 2015;36:299-305.

32 Molinari C, Rizzi M, Squarzanti DF, Pittarella P, Vacca G, Renò F: 1 $\alpha, 25$-Dihydroxycholecalciferol (Vitamin D3) induces NO-dependent endothelial cell proliferation and migration in a three-dimensional matrix. Cell Physiol Biochem 2013;31:815-822

-33 Guo J, Xia N, Yang L, Zhou S, Zhang Q, Qiao Y, Liu Z: GSK-3 $\beta$ and vitamin D receptor are involved in $\beta$-catenin and snail signaling in high glucose-induced epithelial-mesenchymal transition of mouse podocytes. Cell Physiol Biochem 2014;33:1087-1096.

-34 Yu D, Shang Y, Luo S, Hao L: The TaqI gene polymorphisms of VDR and the circulating, 25-dihydroxyvitamin D levels confer the risk for the keloid scarring in Chinese cohorts. Cell Physiol Biochem 2013;32:39-45. 


\section{Erratum}

$\star \star \star *$

In the original article [Cell Physiol Biochem 2015;37:1686-1692 (DOI: 10.1159/000438534)]

Vitamin D Deficiency and Increased Risk of Bladder Carcinoma: A Meta-Analysis

${\text { Zhang } \mathrm{H}^{\mathrm{a}} \quad \text { Zhang } \mathrm{H}^{\mathrm{b}} \quad \text { Wen } \mathrm{X}^{\mathrm{a}} \quad \text { Zhang } \mathrm{Y}^{\mathrm{a}} \quad \text { Wei X }}^{\mathrm{a}} \quad$ Liu T $^{\mathrm{a}}$

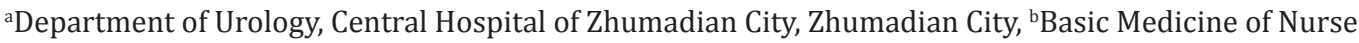
Department, Huanghuai College, Zhumadian City, China

there are few corrections, as following:

- Zhang H. and Zhang H. contributed equally to this work and thus share first authorship

- The correspondig author's address should be changed into:

Hui Zhang

Basic Medicine of Nurse Department, Huanghuai College, Zhumadian City, China

E-Mail53197918@qq.com

The authors terribly apologized for this carelessness. 